\title{
Kommunikation und Emotion
}

In Disziplinen wie der Psychologie und Soziologie ist ein sprunghaft steigendes Interesse am Thema "Emotionen " zu verzeichnen. In der Sprachwissenschaft ist die Analyse der Zusammenhänge zwischen kommunikativen und emotionalen Prozessen dagegen eine nach wie vor vernachlässigte Fragestellung. Wesentlicher Grund hierfür ist, daß die meisten Sprachtheorien Sprache und Kommunikation als vorwiegend kognitive, zweckrationale und instrumentelle Erscheinungen auffassen. Hintergrund dieser Theorie ist das Bild kognitiv geleiteter und rational handelnder Menschen. "Kommunikation als zweckrationaler Austausch von Informationen “ ist aber - wie schon Malinowski sehr plastisch gezeigt hat - eine nur bedingt zutreffende Auffassung, die ein zu kognitiv-rationales Menschenbild zur Voraussetzung hat. Emotionen waren so immer eine "Restkategorie der linguistischen Auffassung von Sprache" (Konrad Ehlich), in die all das hineingepackt wurde, was das zweckrationale Bild störte.

Ziel meiner Arbeit in den letzten Jahren war deshalb, die Rolle von Emotionen in faktischen Gesprächen zu untersuchen und einige der kommunikativen Verfahren und Muster herauszuarbeiten, mit denen Gefühle in der Interaktion dargestellt, gedeutet und bearbeitet werden. Dabei solle zugleich gezeigt werden, daß Gefühle - mehr als wir üblicherweise meinen - sozial geformt und geregelt sind.

Der Begriff Gefühl hat verschiedene Bedeutungen, von denen nur eine das bezeichnet, worum es hier geht. Zum einen kann Gefühl sich auf eine körperliche Empfindung beziehen (Ich hatte kein Gefühl mehr im Bein.). Zum anderen bezeichnet Gefühl häufig einen intuitiven, vagen, nicht belegbaren Eindruck bzw. eine solche Einschätzung. Das dem Sprachwissenschaftler vielleicht naheliegendste Gefühl - das Sprachgefühl - fällt unter diese Bedeutung. Nur die dritte Bedeutung von Gefühl - im Sinne von emotionalem Erleben ist im folgenden relevant.

Emotionen und Gefühle - ich verwende die Begriffe bedeutungsgleich - verstehe ich als spezielle Formen des Erlebens. Der Begriff Erleben umfaßt alle Formen des Erlebens meiner selbst, anderer und der Umwelt. Das, was ich unter Emotionen verstehe, ist Teil des Erlebens, macht aber nicht das ganze Erleben aus. Man kann Ärger, Ekel und Freude, die für mich prototypische Emotionen sind, ebenso erleben wir Irritationen, Unsicherheit, Neugier, Müdigkeit und Hunger, was für mich keine bzw. keine "reinen " Emotionen sind. Im Erlebensprozeß können Emotionen dominant sein, sie können aber auch - und dies ist wohl der Regelfall - in ganz unterschiedlichen Mischungsverhältnissen mit Kognitionen oder mit physiologischen Zuständen auftreten.

\section{Emotionen in uns und in der Interaktion}

Für Emotionen kann man sich aus zwei unterschiedlichen Perspektiven interessieren: Zum einen kann man Emotionen als Elemente eines individuellen Innenlebens betrachten, und man kann fragen, in welchem Verhältnis sie zu anderen Elementen des Innenlebens stehen und wie sie sich Ausdruck verschaffen. Zentral für eine solche Sichtweise sind u. a. die folgenden Annahmen:

(1) Gefühle existieren primär in unserem Inneren.

(2) Gefühle sind etwas Privates. Sie sind individuell. Sie gehören mir.

(3) Gefühle können auf unserer Oberfläche Ausdruck finden und sich so für andere zeigen. Sie können aber auch verborgen oder beherrscht werden.

(4) Gefühle sind ein Widerfahrnis und eine fremde Macht. Sie entstehen ohne unser Zutun. Sie können uns, wenn sie stark sind, beherrschen, d.h. sie sind nicht oder nur schwer kontrollierbar, und sie können uns zu ungewollten Handlungen veranlassen.

(5) Gefühle bilden eine eigene Welt und stehen im Gegensatz zu Verstand und Denken.

Zum anderen kann man sich für Emotionen interessieren als öffentliche Phänomene in sozialen Situationen interpersoneller Interaktion. Das heißt, man fragt nach Funktion und Stellenwert von Emotionsmanifestationen in der Interaktion, unabhängig davon, ob die Beteiligten die manifestierten Emotionen auch empfinden bzw. "wirk- lich « haben. Man interessiert sich dafür, wie Emotionen in der Interaktion wechselseitig manifestiert, gedeutet und gemeinsam "bearbeitet" werden, und letztlich, mit welchen kommunikativen Verfahren und Mustern die Beteiligten dies tun.

Diese beiden Perspektiven sind wie die zwei Seiten der Medaille. Emotionen sind sowohl relevant im Personalsystem wie im Interaktionssystem. Aber man muß sich deutlich vor Augen halten, daß diese beiden Perspektiven einen unterschiedlichen Untersuchungsgegenstand konstituieren, der jeweils anderen Disziplinen zuzuordnen ist der Psychologie bzw. der Interaktionsforschung - und der jeweils andere Verfahren zu seiner Untersuchung erfordert.

Für diesen Beitrag sind Emotionen nur als Phänomene der sozialen Interaktion relevant. Zentrale Annahmen einer solchen Position sind:

(1) Emotionen sind primär als interaktive Phänomene relevant.

(2) Emotionen werden als etwas Öffentliches betrachtet.

(3) Den Emotionsmanifestationen in der Interaktion können, müssen aber keineswegs Emotionen zugrundeliegen.

(4) Emotionen erfüllen primär die Funktion einer bewertenden Stellungnahme. Sie sind ein spezifisches Verfahren und eine spezifische Form der Bewertung.

(5) Emotionen sind geregelt. Die bewertende Stellungnahme erfolgt in weiten Bereichen auf der Grundlage sozial verbindlicher Emotionsregeln.

(6) Emotionen werden in der Interaktion kommuniziert. Dies geschieht in wesentlichen Teilen durch Emotionsmanifestationen und ihre Deutung.

(7) Interaktionsrelevant manifestierte Emotionen können interaktiv weiterbearbeitet werden.

(8) Emotionen sind eine spezifische Form des Erlebens.

(9) Emotionen sind - individuell wie interaktiv - regulierbar.

(10) In der Interaktion wird Erleben auf verschiedenen Stufen der Konkretisierung manifestiert, bis hin zu abgrenzbaren Emotionen.

Ich will im folgenden nur die Punkte (4), (6) und (7) etwas genauer betrachten.

\section{Emotionen als bewertende Stellungnahmen}

In funktionaler Perspektive erfüllt jedes Erleben und jede Emotion die Funktion einer bewertenden Stellungnahme. Mit dem folgenden Schema kann jede konkrete Emotion beschrieben werden:

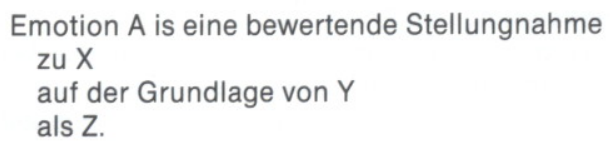

Hierbei sind für $\mathrm{X}, \mathrm{Y}$ und $\mathrm{Z}$ folgende Belegungen möglich:

\begin{tabular}{lll}
\hline \multicolumn{1}{c}{ zuX } & $\begin{array}{l}\text { auf der Grundlage } \\
\text { von } Y\end{array}$ & als Z \\
\hline (1) Situation & (1) Erwartungen & (1) (gut) entsprechend \\
$\begin{array}{lll}\text { (2) andere Person } \\
\text { - Handlung } \\
\text { - Eigenschaft }\end{array}$ & (2) Interessen, Wünsche & (2) nicht entsprechend \\
$\begin{array}{l}\text { (3) eigene Person } \\
\text { - Handlung } \\
\text { - Eigenschaft }\end{array}$ & (3) (akzeptierte) soziale & \\
(4) Ereignis/Sachverhalt & (4) Selbstbild & \\
(5) Gegenstände & (5) Bild des anderen & \\
(6) mentale Produktionen &
\end{tabular}


Ärgere ich mich beispielsweise, weil ich eine Vase umgestoßen habe, so wäre dies eine bewertende Stellungnahme zu der eigenen Person (bzw. einer Aktivität von ihr) auf der Grundlage meines Selbstbildes (bzw. Erwartungen über mein Verhalten) als diesem nicht entsprechend. Freue ich mich bei dem Gedanken, daß ich morgen Besuch bekommen werde, so wäre dies eine bewertende Stellungnahme zu einer mentalen Produktion auf der Grundlage meiner Wünsche (oder Erwartungen) als diesen gut entsprechend.

Wird in der Interaktion eine Emotion kommuniziert, so kann man dies also auffassen als Kommunikation einer solchen bewertenden Stellungnahme oder allgemeiner: als Kommunikation einer Bewertung. Um zu verstehen, welchen Stellenwert die Kommunikation von Emotionen für die Interaktion besitzt, darf man Kommunikation nicht eindimensional oder einaspektig auffassen. Man muß davon ausgehen, daß Kommunikation mindestens zwei prinzipiell gleichrangige Aspekte hat:

- die Verständigung über Sachverhalte und

- die Verständigung über Bewertungen.

Parallel zum Austausch über ein Thema werden immer auch Bewertungen kommuniziert. Ein Teil der Bewertungen, die kommuniziert werden, wird als Kommunikation von Emotionen realisiert.

Der Bewertungsaspekt des Kommunikationsprozesses wurde und wird in der sprachwissenschaftlichen Analyse von Kommunikation systematisch vernachlässigt zugunsten des Informationsaspekts. Aber nur wenn man Kommunikation als unauflösliche Einheit aus Informationen und Bewertungen versteht, wird der systematische Stellenwert der Kommunikation von Emotionen als spezielle Form der Kommunikation von Bewertungen deutlich.

\section{Emotionen und kommunikative Prozesse}

Fragt man, wie Emotionen in kommunikative Prozesse eingelagert sind, oder von der Gegenseite aus betrachtet, wie Kommunikation in emotionale Prozesse integriert ist, so lassen sich drei wesentliche Bezüge unterscheiden: Kommunikative Prozesse (bei) der Manifestation, der Deutung und der Weiterverarbeitung von Erleben und Emotionen.

Wenn Personen miteinander interagieren, so manifestieren sie ihr Erleben und ihre Emotionen in sehr verschiedenen Verhaltensbereichen. Sprachliche und kommunikative Möglichkeiten der Manifestation stellen dabei einen Spezialfall mit spezifischem Anteil und speziellen Funktionen dar. Ich unterscheide zwei Gruppen von Verfahren der Manifestation: einerseits Ausdruck und andererseits Thematisierung von Erleben und Emotionen.

Während bei der Thematisierung ein Erleben oder eine Emotion durch eine Verbalisierung zum Thema der Interaktion gemacht wird, sind Ausdrucksphänomene nicht an Verbalisierungen gebunden (sie können sie aber natürlich begleiten), und sie machen das mit ihnen manifestierte Erleben nicht (notwendig) zum Thema der Interaktion. Ein unwirsches Kannst du vielleicht auch mal pünktlich sein? bringt zwar durch Intonationskontur, Stimmcharakteristika, Sprechgeschwindigkeit etc. ein Erleben zum Ausdruck, thematisiert es aber nicht, wie es mit Du machst mich ganz ärgerlich. Ewig diese Unpünktlichkeit! der Fall ist. Das emotionale Erleben kann also - wie tausend andere Dinge auch - Thema der verbalen Kommunikation sein. Wir kommunizieren dann über Emotionen. Dies ist die Grundstruktur der Thematisierung von Emotionen und Erleben.

Meistens ist aber ganz etwas anderes das Thema der verbalen Kommunikation. Wir kommunizieren über etwas anderes, aber daneben und zugleich kommunizieren wir - durch die Art, wie wir über das Thema kommunizieren - Emotionen. Sie haben die Funktion bewertender Stellungnahmen zum Thema, aber auch zu weiteren Aspekten der Situation: zu anderen Personen, zu ihren Handlungen, zu uns selbst etc. Dies ist die Grundstruktur des Ausdrucks von Emotionen und Erleben.

Auch für die Erlebensthematisierung existieren wieder verschiedene kommunikative Verfahren, u.a. die begriffliche Erlebensbenennung und die Erlebensbeschreibung.

Die begriffliche Erlebensbenennung erfolgt mit Elementen des Erlebenswortschatzes. Erlebensbegriffe sind sozial vorgeformte und normierte Deutungsmöglichkeiten für individuelles Erleben. Zum Erlebens- und Emotionswortschatz liegt eine Vielzahl von Untersuchungen vor. Fast noch interessanter, aber kaum untersucht sind hingegen die Erlebens- und Emotionsbeschreibungen.

Diese sind mehr oder minder ausführliche Versuche, ein spezifisches Erleben dem Interaktionspartner durch Umschreibungen zu verdeutlichen. Viele der Erlebensbeschreibungen erfolgen mit Vergleichen und bildlich-metaphorischen Mitteln. Über Erleben und
Emotionen läßt sich kaum anders reden, als daß sie in Analogie zu anderen (konkreteren) Bereichen konzeptualisiert werden. Dabei handelt es sich häufig nicht um einzelne Analogien, sondern bestimmte Bereiche sind die Quelle für eine Vielzahl bildlicher Erlebensbeschreibungen. Einige wesentliche und produktive Bereiche möchte ich kurz benennen:

Negatives Erleben wird häufig konzeptualisiert als körperliche Verletzung oder Störung der körperlichen Integrität: etwas hat mich unheimlich verletzt/gekränkt, es hat geschmerzt, Wunden geschlagen, Narben zurückgelassen, etwas nagt in mir, ich zerfleische mich, etwas trifft mich wie ein Stich ins Herz oder geht unter die Haut. Erleben und Emotionen werden ferner konzeptualisiert als Sinneswahrnehmung: das schmeckt mir nicht, das stinkt mir, ich kann ihn nicht riechen, das kratzt mich nicht. Weitere Konzepte sind Hitze und Kälte (das ließ mich kalt, es kocht in mir), der bis Explosion ansteigende Druck (es zerriß mir das Herz, ich explodierte) und das Abheben vom Boden (wer wird denn gleich in die Luft gehen, du treibst mich auf die Palme). Zwischen diesen Konzepten bestehen enge Beziehungen, und sie gehen über in Konzepte, mit deren Hilfe Emotionsdynamik beschrieben wird: Steigen und Fallen von Wasser (ein Gefühl von Angst über-/durchflutete mich, dann verebbte es), Entflammen und Brennen sowie Wind und Sturm.

Positive Gefühle werden u.a. als Gewichtsverlust konzeptualisiert, der es ermöglicht, zu schweben oder zu fliegen: mir ist ein Stein vom Herzen gefallen, was für eine Erleichterung, ich schwebte wie auf Wolken, fühlte mich im siebten Himmel. Dies ist ein Spezialfall von Konzeptualisierungen, die die Dimension Höhe/Tiefe benutzen, wobei positiv bewertete Entitäten hoch und negativ bewertete tief sind (ich bin down, am Boden zerstört).

Analysiert man auf diese Weise den Gesamtbereich der in Erlebensbeschreibungen benutzten Bildlichkeit, so lassen sich durchaus grundlegende, in unserer Kultur übliche Auffassungen über Emotionen ableiten. Es handelt sich dabei um elementare kognitive Konzepte, die kulturspezifisch unsere Vorstellungen von Emotionen und inrer Funktionsweise strukturieren und bestimmen.

\section{Bearbeitung von Emotionen und Erleben}

Sind Erleben und Emotionen durch Manifestation und Deutung für die Beteiligten als gemeinsames Faktum etabliert, müssen sie in der Interaktion bearbeitet ("prozessiert") werden. Dabei lassen sich analytisch vier Strategien unterscheiden: Die Strategie Eingehen umfaßt alle Formen, bei denen der Interaktionspartner die manifestierte Emotion als angemessen akzeptiert und mit Bekundungen der Anteilnahme auf sie eingeht. Hinterfragen bezeichnet zusammenfassend Interaktionsverläufe, in denen die Angemessenheit der manifestierten Emotionen in Hinblick auf Intensität und/oder Art problematisiert wird. Beim Infragestellen wird das manifestierte Erleben nicht als angemessen akzeptiert. Beim Ignorieren schließlich vermeidet der Interaktionspartner bewußt und offensichtlich, obwohl er es wahrgenommen und gedeutet hat, auf das Erleben einzugehen und es in manifester Weise interaktiv zu behandeln. Sein demonstrativer Charakter unterscheidet das Ignorieren vom Übergehen. Diese Strategien können auch kombiniert auftreten. Offensichtlich ist, daß die drei erstgenannten an verbale Kommunikationsprozesse gebunden sind.

Im Rahmen dieser Strategien sind einige Formen der Behandlung von Emotionen zu kommunikativen Mustern verfestigt. Muster sind sozial standardisierte und konventionalisierte Verfahren, die zur Realisierung spezifischer, im sozialen Prozeß häufig wiederkehrender Aufgaben und Zwecke dienen. So ist z. B. für die Strategie "Eingehen " das Anteilnahmemuster zentral, für die Strategien "Hinterfragen «/»Infragestellen " ist es das Divergenzmuster. Mit seiner Hilfe wird eine manifeste Emotion, sofern sie vom Partner nicht akzeptiert wird, Gegenstand einer Aushandlung. Das Divergenzmuster möchte ich an zwei Beispielen veranschaulichen:

\section{(1) A: Wie kannst du so lustig sein, wo du doch gerade eine Fünf geschrieben hast? \\ B: Du hast recht, ich habe wirklich keinen Grund dazu.}

A bezeichnet Ausdrucksphänomene bei B zusammenfassend als lustig sein. Die Aushandlung ist gerichtet auf eine Veränderung des hinter diesen Phänomenen stehenden Erlebens, das nicht den für die Situation geltenden Emotionsregeln entspricht und das qualitativ verändert werden soll. Die sanktionierende Funktion überwiegt. Es handelt sich um einen Fall interaktiver Emotionsregulation. Mit der Formulierung keinen Grund anerkennt B, daß sein Erleben und das 
entsprechende Ausdrucksverhalten nicht durch Emotionsregeln gedeckt ist.

(2) A: Seine blöden Bemerkungen hätte sich $C$ auch sparen können! B: Ärgere dich doch nicht so. Das ist es doch nicht wert.

A: Laß mich doch. Ich will mich nun mal aufregen.

B deutet A's Ausdrucksphänomene (z.B. Wortwahl, Intonation) und benennt sie als Ärger. B's Vorschlag im Rahmen der mit seiner Äußerung einsetzenden Aushandlung ist auf eine Veränderung der Intensität des Erlebens (so) gerichtet. Der Vorschlag zur Veränderung des Erlebenszustandes wird in Form einer direkten Aufforderung realisiert. Die Begründung rekurriert auf eine Emotionsregel, wonach eine hohe Intensität des Erlebens an eine hohe Bedeutsamkeit des Ereignisses gekoppelt ist. Die korrigierende Funktion des Vorschlags überwiegt. Der Versuch interaktiver Emotionsregulation wird aber von A zurückgewiesen, wobei allerdings eine andere Erlebensbenennung (aufregen) verwendet wird.
Die Beispiele belegen, daß über Emotionen sehr wohl "verhandelt “ werden kann und daß dies nicht nur die Manifestationen, sondern auch die Emotionen selbst betreffen kann. Solche Aushandlungen werden auf der Grundlage impliziter oder explizit angeführter Emotionsregeln ausgetragen. Über Gefühle wird dabei argumentiert auf der Grundlage von Vorstellungen wie: eine Emotion ist "angemessen/unangemessen “, sie besteht "zu Recht/zu Unrecht “, sie ist "begründet/unbegründet " etc. Aushandlungen und die zugrundeliegenden Emotionsregeln machen ein weiteres Mal deutlich, daß Gefühle über weite Strecken keine "Privatsache ", sondern in der Tat sozial normiert sind.

Literaturhinweis

Fiehler, Reinhard: Kommunikation und Emotionen. Theoretische und empirische Untersuchungen zur Rolle von Emotionen in der verbalen Interaktion. Berlin: de Gruyter 1990.

Der Autor ist Privatdozent für Sprachwissenschaft an der Universität Bielefeld. 